\title{
Foci determination of an off axis ellipsoid: From theory to practice
}

\author{
Y. Houbrechts, S Roose, Y. Stockman \\ Centre Spatial de Liège, Avenue du Pré-Aily, B-4031 Angleur-Liège, Belgium.
}

\begin{abstract}
In the frame of cryogenic test on an off axis ellipsoid, it has been required to set-up an unambiguous method to determine and track the foci position during temperature transitions. This procedure was mandatory to avoid:

- impact of the operator skills working on triple shift scheme to assure continuously monitoring of the ellipsoid shape during cool down

- correctly dissociate the impact of the thermal deformation on the mirror shape with respect to alignment errors.

This paper will demonstrate the process, starting from ideal ellipsoid shape, then introducing 3D metrology data in a model, and finally presents the results in a practical situation.
\end{abstract}

KEYWORDS: non-destructive testing, non-standard surfaces and quality control, correlation between experiment and theory.

\section{INTRODUCTION}

The present reflector to be tested is a CFRP off-axis ellipsoids. The goals of the tests are to measure the modification of the surface figure error (SFE) with respect to the best ellipsoid, between $293 \mathrm{~K}$ and cryogenic temperatures, with a $1 \mu \mathrm{m}$ RMS accuracy. The second objective is the focus position change measurement with accuracy of $20 \mu \mathrm{m}$ during the temperature variations.

Preliminary assessments have shown that only few practical methods can be used to measure both SFE and track the focus changes at the same time. CSL proposed to employ a wave-front measurement method [1], to be operated in stigmatic configuration as presented in Figure 1.

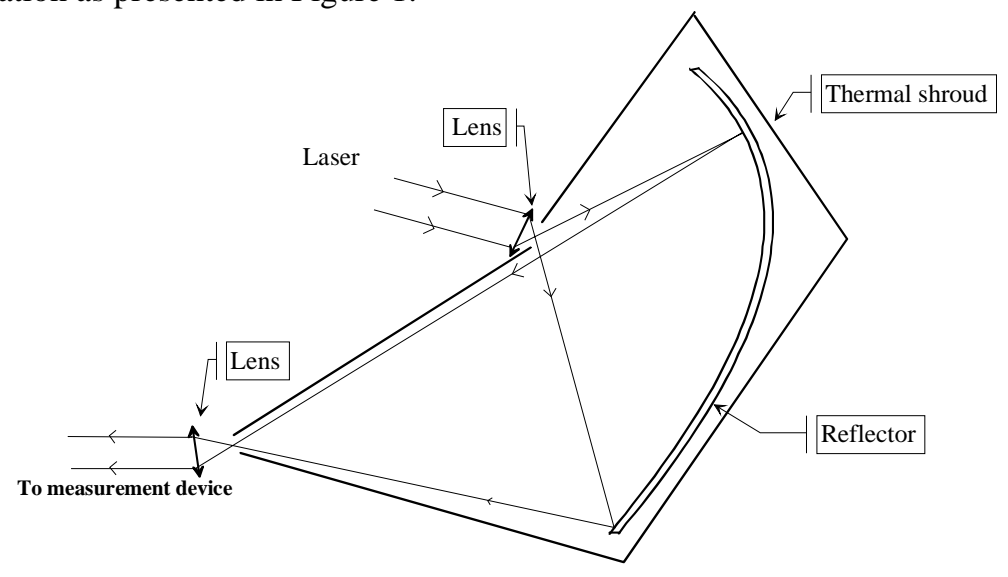

Figure 1 : Stigmatic test configuration used for reflector test (single pass). 
This paper will concentrate on the procedure developed to evaluate the foci determination and their displacements during temperature variations. After a presentation of the test set-up and the facility, the procedure is presented in the case of a perfect ellipsoid, without SFE. The set-up is modelled with ASAP ${ }^{\mathrm{TM}}$ software. With this model, it is demonstrated that it is possible to recover the best focus position starting from an unknown position. The only requirement is to get resolvable fringe density in the interferogram. The next step consists to introduce the SFE of the real reflector measured with a 3D metrology machine. Again, it appears to be possible to recover the focus position starting from unknown positions. Finally, the method is faced to the real world and demonstration is performed that the focus changes can be tracked by the proposed method on the experimental set-up. The encountered difficulties are highlighted and the way to solve them is presented.

\section{TEST SET -UP AND FACILITY}

The FOCAL 3 for Facility for Optical Calibration At Liége is a $3 \mathrm{~m}$ diameter vertical vacuum vessel (Figure 2). The level of pressure reached in the space simulation chambers is lower than 10-3 Pa. This chamber is located in a class 10000 clean room. The FOCAL 3 facility included an optical table attached to a seismic bloc. All the optical parts are linked to the massive seismic bloc that attenuates the surrounding vibration. The reflector (Figure 3 ) is surrounded by a double thermal tent, the external one is fed with liquid Nitrogen, while the internal one is fed with liquid He. These thermal tents were specially shaped to surround the reflector, and to let the optical part of the set-up, which is not under test at ambient temperature. As it will be explained latter, due to the position of one focal point in the shadow of the other it is not possible to avoid small vignetting by the shrouds (see Figure 7) of the reflector aperture.

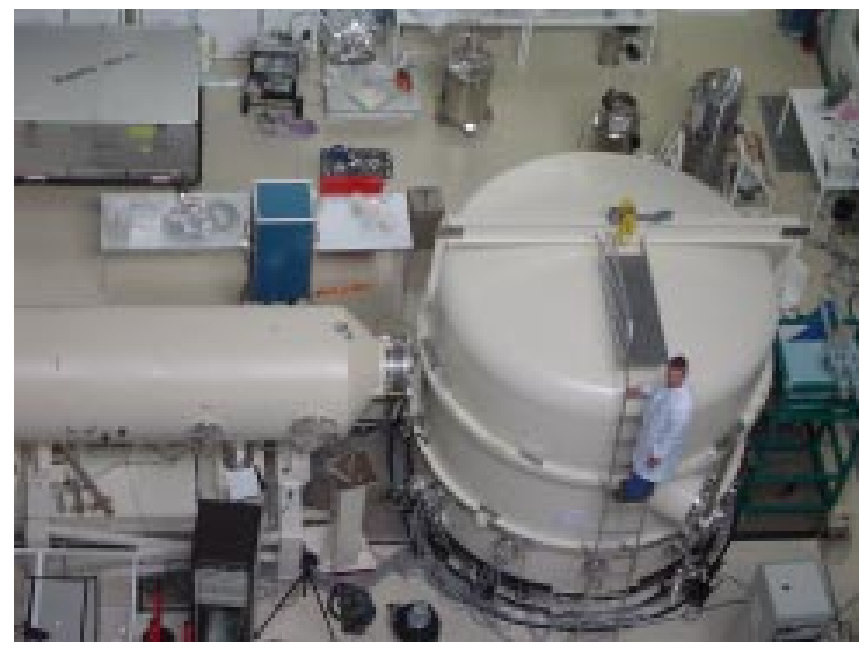

Figure 2 : FOCAL 3 CSL facility

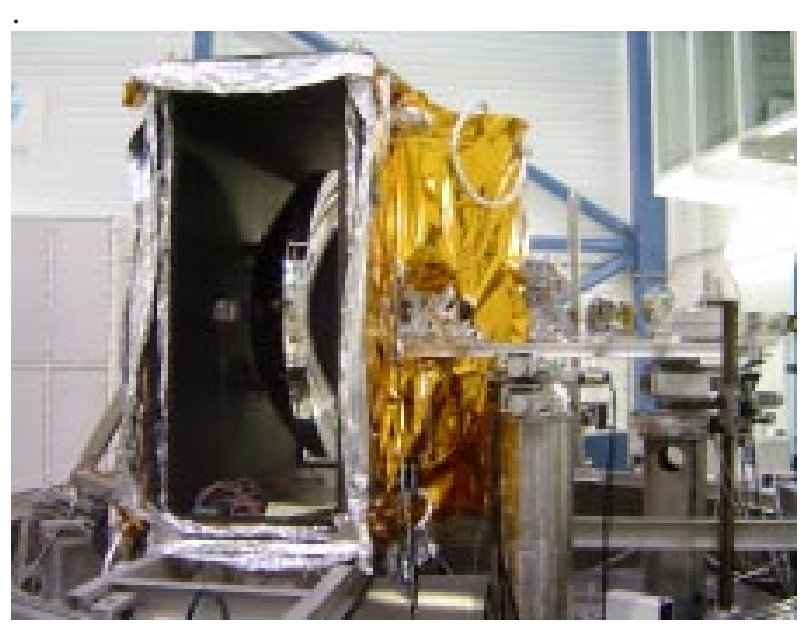

Figure 3 : Reflector in its thermal shrouds

\section{OPTICAL TEST CONFIGURATION}

The reflector is a $1050 \mathrm{~mm} \times 1100 \mathrm{~mm}$ CFRP dish. The primary focus is about $360 \mathrm{~mm}$ from the reflector, and the secondary focus is at $720 \mathrm{~mm}$. The reflector surface has a good reflectivity in the visible and IR. However, the micro roughness is $\mathrm{Rq}<0.2 \mu \mathrm{m}$ RMS at any scales up to $0.8 \mathrm{~mm}$. This obliges us to work with infrared light in order to limit scattering. Moreover the SFE slopes are very high and some parts of the reflector would not allow an interferometric measurement with visible light. Indeed a standard detector would not resolve the high interferometric fringe density associated to high surface slopes

As presented in Figure 1, the test configuration is a single pass interferometric configuration.

The interferometer used is a commercial instrument manufactured by Breault Research Organisation (BRO). This one has already been used at CSL to test several IR antennas [2] and at COI to test FIRST antenna [3]. Unfortunately, since a couple of years this interferometer is not more supported by BRO. Only the mainframe and some optics have been keep, the remainder hardware and software had to be updated. This interferometer is an Infra Red Phase Shifting Interferometer (IR PSI). The original CO2 laser source working at $10.6 \mu \mathrm{m}$ has been replaced. The interferometer cavity including the piezo system, beam splitter and compensating wedge have been taken out of the mainframe and put inside 
the vacuum chamber but outside the cryogenic tent. A dedicated piezo driver substituted the old one. The piezo can be actuated with a ramp or by steps. The pyro-electric vidicon camera has been replaced by a CEDIP uncooled microbolometer camera of $320 \times 240$ pixels. The CEDIP detector sensitive area is close to the vidicon one, but it was not possible to put it inside the mainframe. Small mechanical modification have been undertaken (Figure 4) to allow the camera integration.

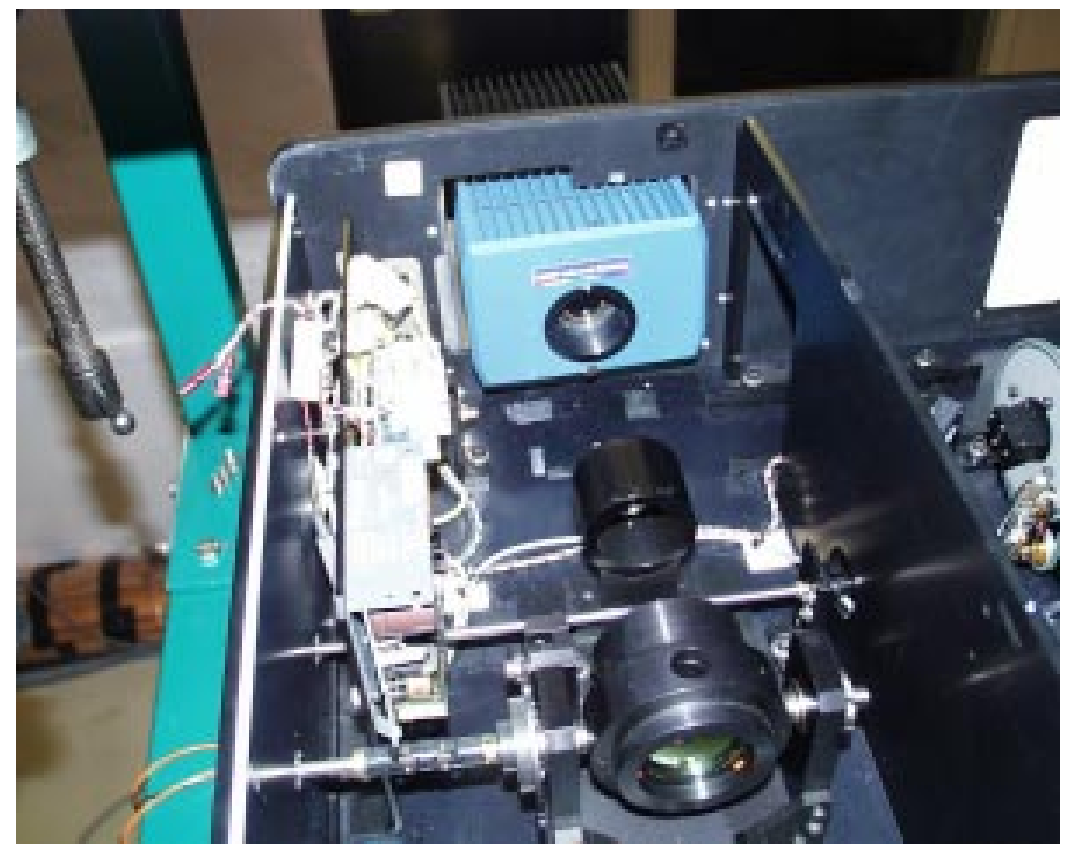

The advantage of this new camera is to have adjustable gain that allows good contrast. Additionally, it works in real time i.e. it does not need to ramp the piezo of the reference mirror as it is requested for the pyro-electric vidicon detector. The drawback is that all the static stray-light is recorded either if it is not part of the modulated signal. All the acquisition and data processing systems were first home made, and updated finally with the Intelliwave $\mathrm{e}^{\mathrm{TM}}$ software from Engineering Synthesis Design. Phase maps where computed from 5 interferometric maps shifted by $90^{\circ}$ with a Bucket 5B. The optical path difference maps are computed with a Minimum Discontinuity unwrapping algorithm. The Figure 4 presents the mainframe after modification.

Figure 4 : IR PSI mainframe after modification

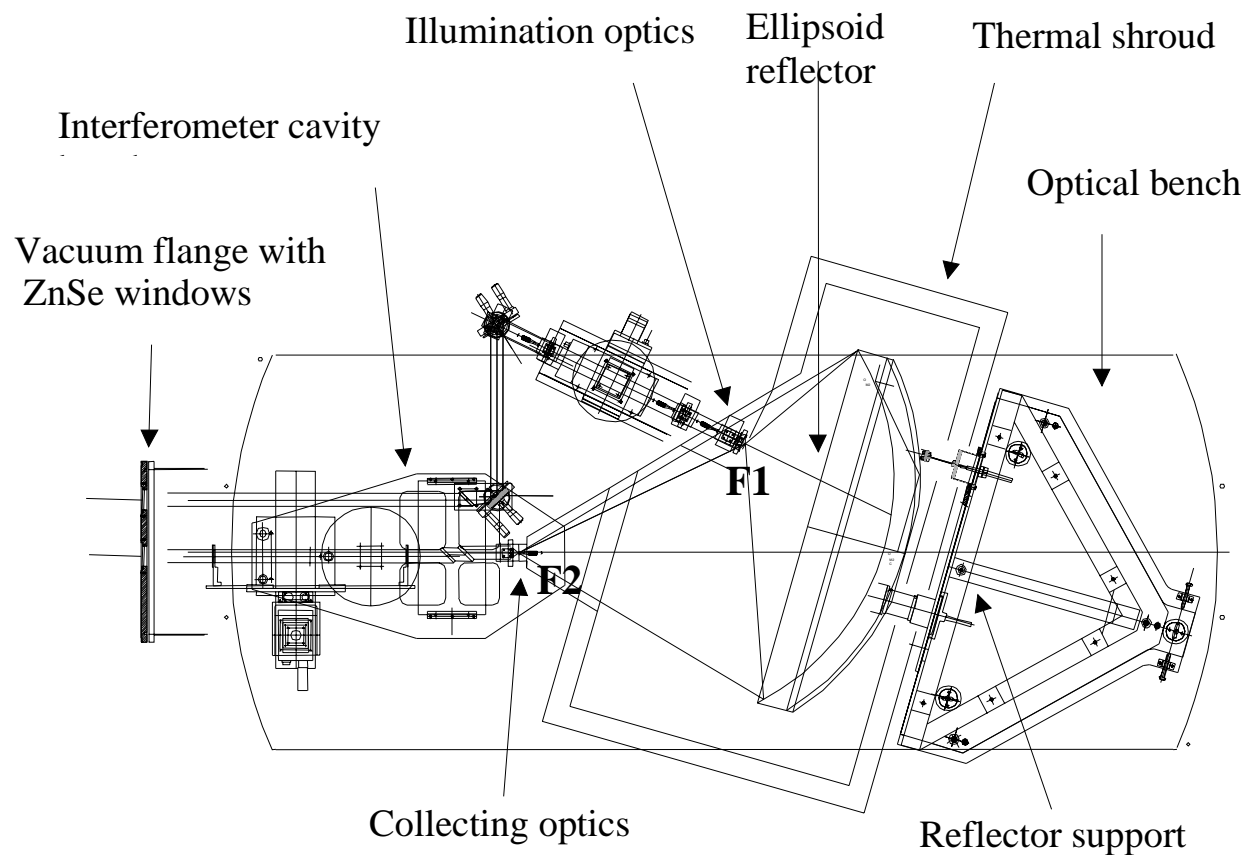

Figure 5 : IR optical test configuration 
The Figure 5 shows the detailed vacuum optical set-up. The mainframe including camera and laser (not on Figure 5, behind the vacuum flange) is outside the vacuum chamber. Light coming out the mainframe crosses a ZnSe wedged window and it is folded by a periscopic system to the focus with smallest F-number (F/0.33) (illumination optics). This optics, including a relay optic and an aspheric ZnSe lens, is mounted on a "3 Degrees Of Freedom" support for positioning of the lens in focus (F1). At the secondary focus (F2) of the reflector (largest F-number (F/0.66)), the light is collected by a spherical lens (collecting optics). An imaging system is specially designed to image the reflector on the interferometer camera.

The selection of this optical configuration is lead by optical design complexity: the lens with the smallest F-number has no field while the lens with highest F-number has to support the reflector field aberrations. All the optical components are mounted outside the cryogenic box. By design, the residual field aberration (only field aberrations are relevant for our measurements) in the system due to the auxiliary optics is better than $0.33 \mu \mathrm{m}$ RMS WFE. The double shrouds system is represented as well as the optical path. It appears clearly that a part of the reflected beam is vignetted by the shrouds in F1.

\section{PRE-ALIGNMENT TOOLS}

We first pre-align the optical set-up with the help of an alignment tool. It consists of two spheres which centres are located at the F1 and F2 positions of the reflector, with an uncertainty of $2 \mathrm{~mm}$ with respect to the nominal foci F1 and F2 positions. These spheres are mounted on a mechanical interface identical to the reflector interface. A first exercise consists in an interferometric alignment of the F1 and F2 lenses in an auto-collimation set-up. The spheres act as autocollimation mirrors and the focal points of the F1 and F2 lenses are aligned with respect to the sphere centre of curvature.

Once the reflector is put in place, the first issue is to get fringes. This is generally straightforward, because the alignment tool is fairly close to the stigmatic foci position. The fringe density is reduced by moving F1 and F2 without any mathematical processing. Once the operator estimated that the best fringe pattern is obtained, the X, Y, Z positions of F1 and F2 are recorded. The $\mathrm{Z}$ axis is the optical axis of the set-up, while the $\mathrm{X}$ and $\mathrm{Y}$ are the two other orthogonal axes.

\section{ALIGNMENT RATIONALE}

In order to understand the alignment rationale one imagines a perfect ellipsoid as an optical imaging system. The F1 optics is the source F1', the F2 optics is the detector F2' and the rule of the game is to put F1' and F2' in the position F1 and F2. The approach is related to the discrimination of some types of aberrations. Indeed, when the detector (interferometer) is moved away from its nominal position it creates low order aberrations (tilt and defocus). When F1' (the source) is not in its position its creates also high order aberrations.

\begin{tabular}{|l|l|l|c|}
\hline $\mathrm{F} 1=\mathrm{F} 1^{\prime}$ & $\mathrm{F} 2 \neq \mathrm{F} 2^{\prime}$ & Tilt and defocus & No high order aberrations \\
\hline $\mathrm{F} 1 \neq \mathrm{F} 1^{\prime}$ & $\mathrm{F} 2=\mathrm{F} 2^{\prime}$ & Tilt and defocus & High order aberrations \\
\hline $\mathrm{F} 1 \neq \mathrm{F} 1^{\prime}$ & $\mathrm{F} 2 \neq \mathrm{F} 2^{\prime}$ & Tilt and defocus & High order aberrations \\
\hline F1=F1' & F2=F2' & No tilt no defocus & No high order aberrations \\
\hline
\end{tabular}

Table 1 : Impact of foci misalignments with respect to aberration types

Its is well known that high order aberrations can be described by a polynomial set. One of the most popular (and widely available in optical software packages) is the Zernike polynomial set.

In this polynomial expansion, there is a dominant term, the one with the highest sensitivity. In the proposed approach, the dominant terms for every degree of freedom in the set-up are identified and a sequence of minimisation of the high order aberrations (WFE), tilt and defocus is established.

The evolution of these Zernike coefficients is studied here after starting from a model with a perfect ellipsoidal reflector. Afterwards the real world reflector is introduced. 


\section{THE SET UP MODELISATION}

To start the study, the complete model of the set up is introduced in $\mathrm{ASAP}^{\mathrm{TM}}$ software. Figure 6 represents a sketch of the set-up. All the lenses have been designed at CSL using a CODE $\mathrm{V}^{\mathrm{TM}}$ software. The IR interferometer optics has been modelled by ideal lenses.

The model takes also into account the actual motor displacements, which are not necessary co aligned with respect to the ellipsoid axes. For analysis, $151 * 151$ rays are send through the system. It is checked that this number of rays is large enough and that the results are relevant with respect to larger number of rays and resolution images. The OPD are computed and saved in a data file. From this OPD data file, the Zernike coefficients are computed.

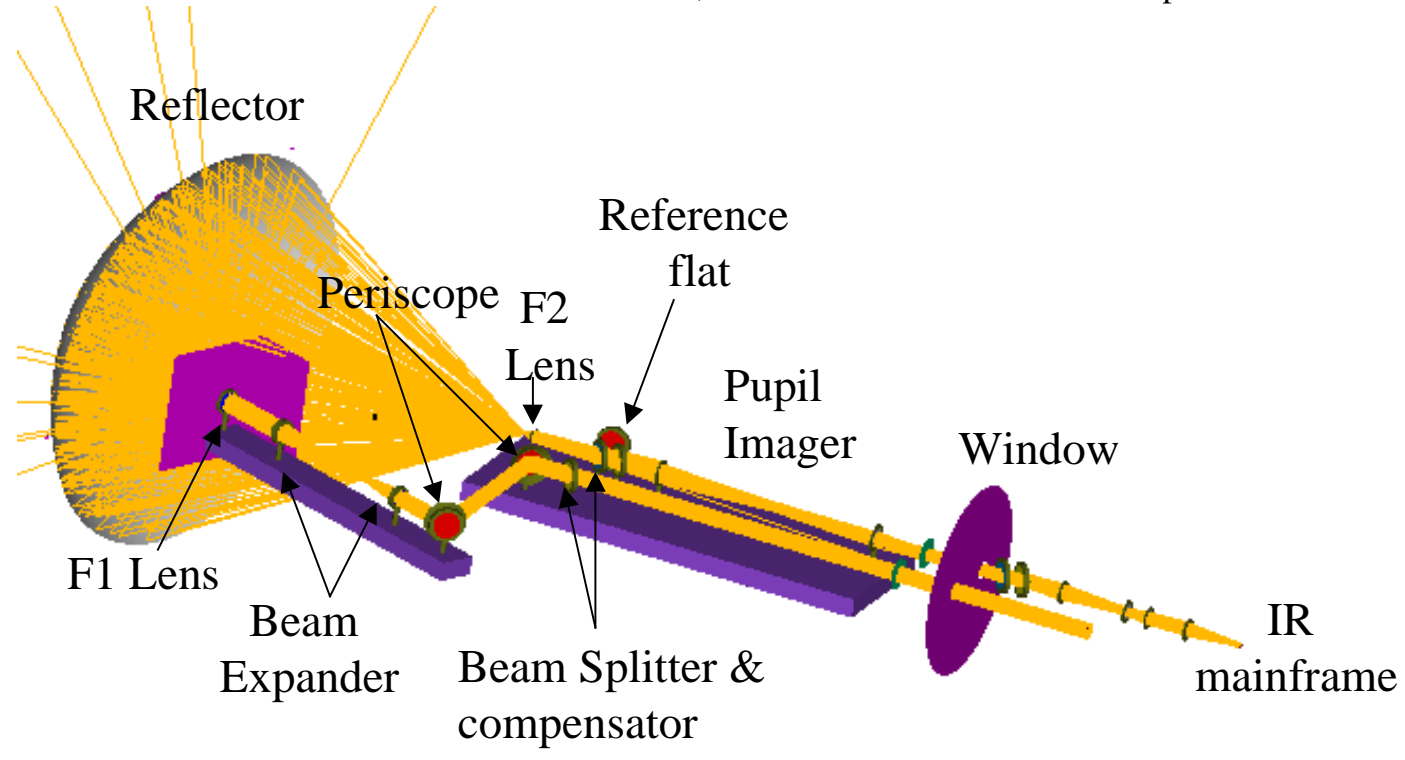

Figure 6 : ASAP model

\section{PROCEDURE DEVELOPMENT FOR A PERFECT ELLIPSOID}

The basic idea of the alignment philosophy is to study the behaviour of the Zernike coefficient with respect to displacement of each focal point. First, it is assumed that the high speed focus (F1) is correctly aligned. If this hypothesis is achieved, the major aberrations, when moving F2 focus, are tilt and defocus. This is easily observed in Figure 9 and Figure 10 where the tilt and astigmatism aberrations are plotted versus lateral displacement of the F2 focus. Only the tilt aberration is sensitive, the astigmatism as well as other aberrations do not change. The tilt curve crosses the 0 tilt when the misalignment of X F2 and Y F2 are 0. Same conclusions can be made with the focus Zernike term when moving the $\mathrm{Z} F 2$ axis. From the simulated data, a regression line is computed, such that the zero aberration position can be computed. The minimum focus and tilt aberrations (equal 0) are achieved when the X, Y, Z F2 are equal to 0 , i.e. the best alignment.

The same basic idea is developed for the determination of the best focal point F1. Obviously, when moving F1, tilt and focus are also changing. Nevertheless, other high order aberration coefficients change too. Astigmatism has been identified as coefficient having the highest sensitivity for lateral X, Y F1 displacement as can be observed on Figure 11. A linear regression is computed, and the position where the astigmatism is zero can be found. Additionally, the $\mathrm{X}$ and $\mathrm{Y}$ axes are nearly independent in term of X and Y Zernike coefficients. Y is co-aligned with the ellipsoid Y axis, while X and $\mathrm{Z}$ translations units are not exactly along the $\mathrm{X}$ and $\mathrm{Z}$ axes of the ellipsoid. 


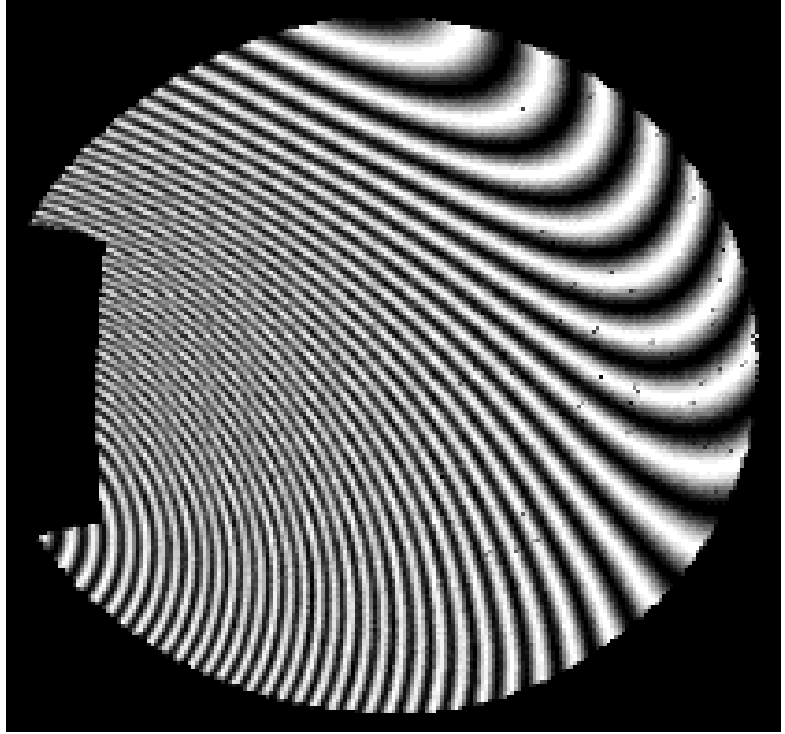

Figure 7 : Simulated interferogram corresponding to a F2 misalignment of $\quad-2.45 \quad 0.26-0.31 \mathrm{~mm}$ and $\mathrm{F} 1 \quad 0.31$ -0.2950 .115 in $\mathrm{X}, \mathrm{Y}, \mathrm{Z}$ direction respectively

\section{Zernike coefficients evolution versus F2X}

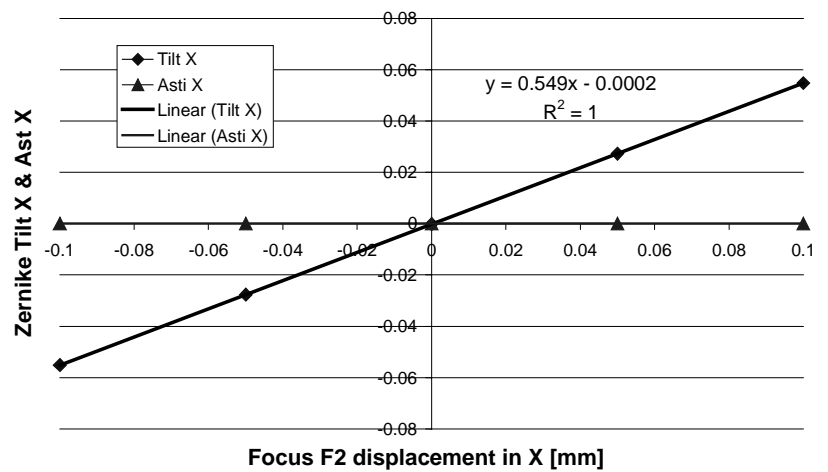

Figure 9: Evolution of the Zernike $X$ tilt and $X$ astigmatism coefficients for different $F 2 X$ positions.

Zernike Astigmatism Y coefficient evolution versus F1 Y

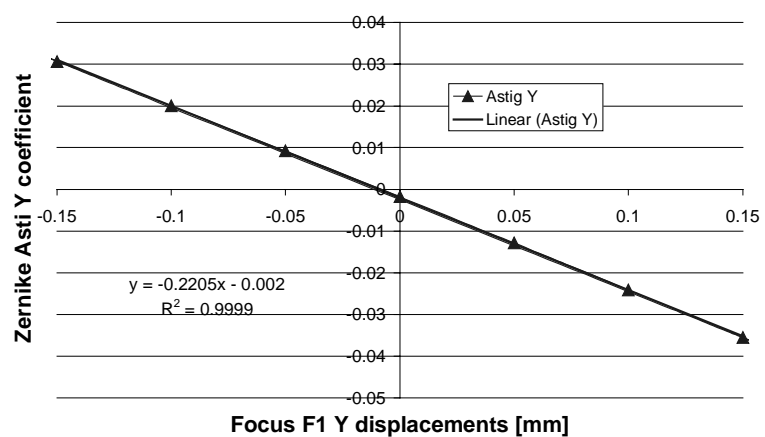

Figure 11: Evolution of the Zernike $Y$ astigmatism coefficient for different F1 Y positions. Same behaves for displacement of F1 Y for Y astigmatism Zernike coefficient

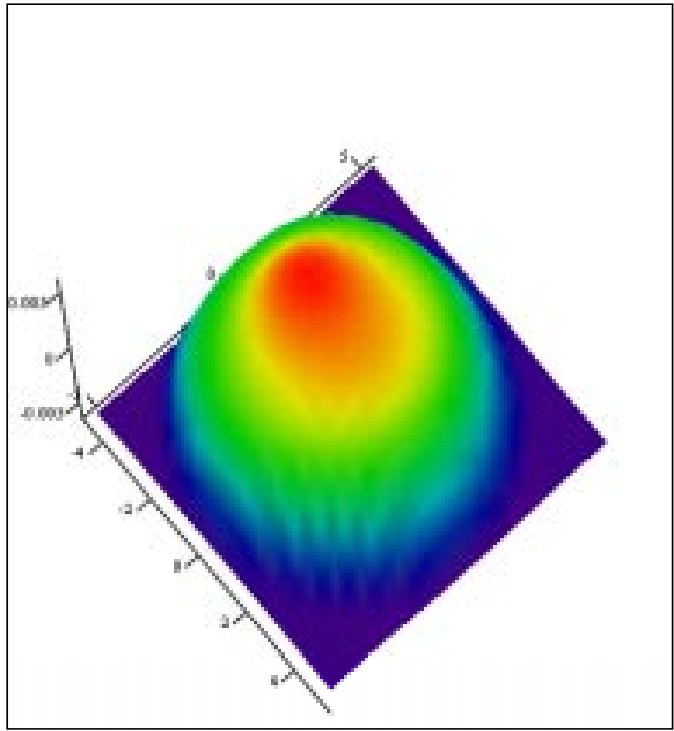

$(\mathrm{x}, \mathrm{y}, \mathrm{zcalc})$

Figure 8 : Computed OPD from the evaluated Zernike coefficient for F2 misalignment of $0.050 .050 .2 \mathrm{~mm}$ in $\mathrm{X}, \mathrm{Y}, \mathrm{Z}$ direction respectively

Zernike Focus coefficient evolution versus F2 Z

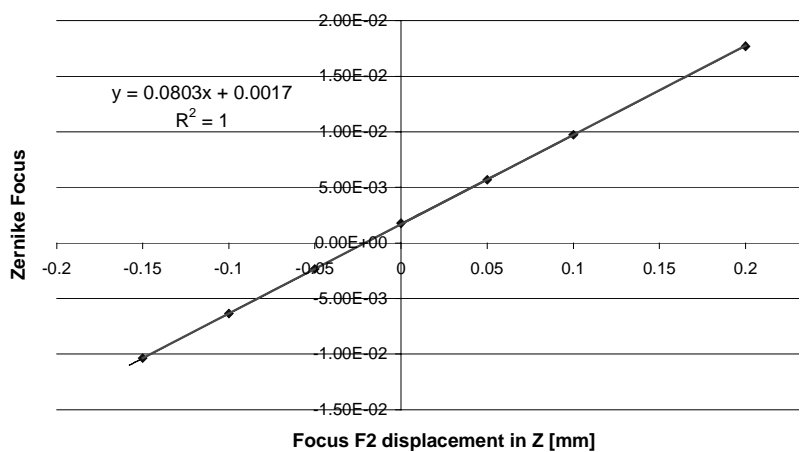

Figure 10: Evolution of the Focus Zernike coefficient for different $F 2 Z$ positions.

RMS variation evolution versus F1 Z

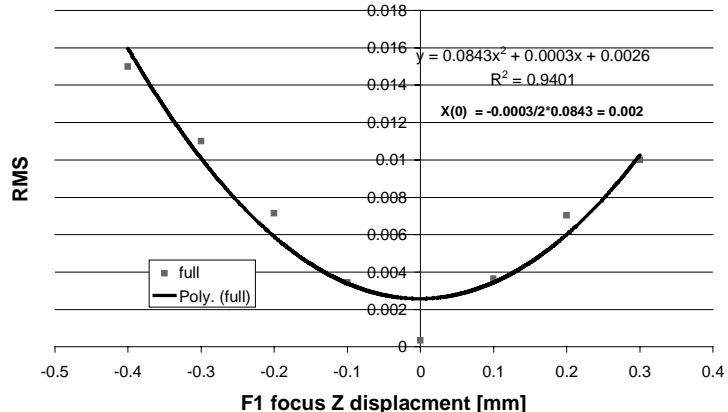

Figure 12: Evolution of the RMS coefficient for different $F 1 \mathrm{Z}$ positions 
For what concerns the focus $\mathrm{Z}$ position of F1, the selected parameter is the RMS wave-front error (without tilt and focus). There was no clear dominant aberration term. Aligning F1 consists in practice in multivariate minimisation of cost-function: the RMS WFE (without tilt and defocus). Since X, Y F1, it is justified to fit the WFE in function of Z F1. The best focus position Z F1 corresponds to the minimum RMS WFE (without tilt and defocus).

From Figure 10 to Figure 12, it can be observed that Zernike coefficient zero is not achieved exactly at 0 but a few $\mu \mathrm{m}$ from this. However, it should be keep in mind that the full set-up is introduced in our model, and that small residual aberrations leave inherent to the optimisation that as been performed. Additionally when optimising F1, F2 is not at the correct position.

In conclusion, the proposed procedure is the following :

Starting from an unknown initial position, the positions X and Y F1 are determined using the linearity behaviour between these displacements and the astigmatism coefficients. Once that the zero $\mathrm{X}$ and $\mathrm{Y}$ astigmatism positions are determined, the system is moved to these positions. The next step is to set the best $\mathrm{Z} \mathrm{F1}$ position by minimising the RMS WFE. These steps concern the F1 focus position. For F2, first the tilt X and Y are corrected by scanning F2 X and $\mathrm{Y}$. Finally, the best focus position is achieved by scanning the $\mathrm{Z} \mathrm{F} 2$ and determining the 0 Zernike focus term $\mathrm{Z}$ position. All the simulated tests show a fast converging to the best focal points (F1 and F2).

\section{THE SIMULATED PROCEDURE APPLIED TO THE REAL CASE}

The proposed procedure looks very promising using an ideal surface. However, before having the hardware operating, the procedure is checked using 3D data (Figure 13) available from the 3-D measurements of the SFE of the real reflector. This allows to bring in the expected reflector shape in the model. The Figure 14 shows a simulated interferogram obtained with the model, once the measured shape of the reflector is introduced. The model is able to compute the residual error (Figure 15) for any type of position of the illumination (F1) and collecting (F2) optic positions. As it can be observed in the next figures (Figure 16 to Figure 19), the aberrations versus focus displacements have the same behaviour as in the ideal case. The propose procedure works well. The final achieved positions are not exactly the same. It comes from the fact that introduced deformed ellipse has not the same stigmatic points as the perfect one. The way the procedure is set up plays on the WFE aberration minimisation, so that some surface defaults are compensated by alignment. Nevertheless, the information coming out of the alignment is the relevant one needed for the end-user.

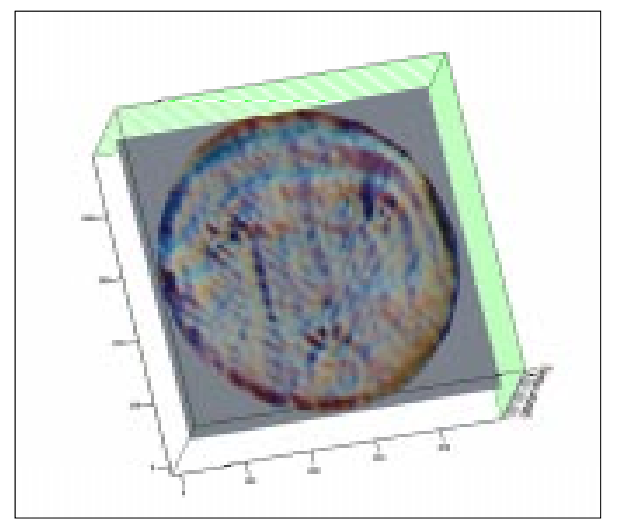

Figure $13: 3 \mathrm{D}$ view of the metrology data

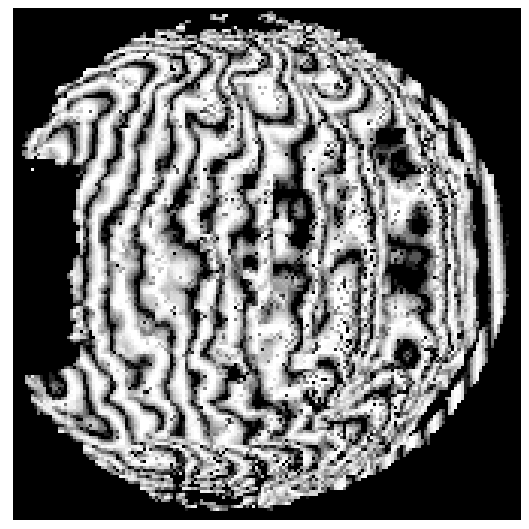

Figure 14: Simulated interferogram including the 3D shape measurement

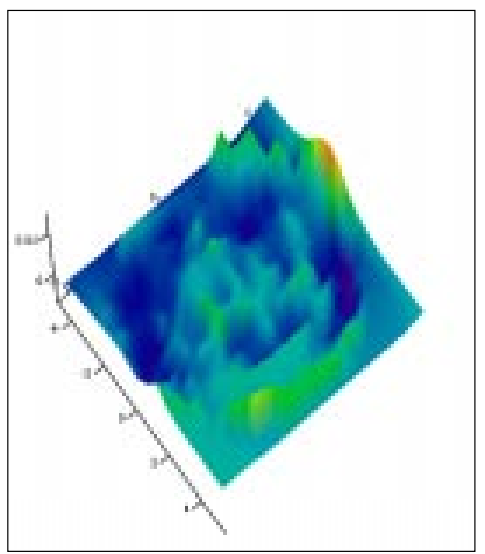

$(\mathrm{x}, \mathrm{y}, \mathrm{residu})$

Figure 15: Computed residual error 
Zernike Astigmatism Y coefficient evolution versus F1 Y

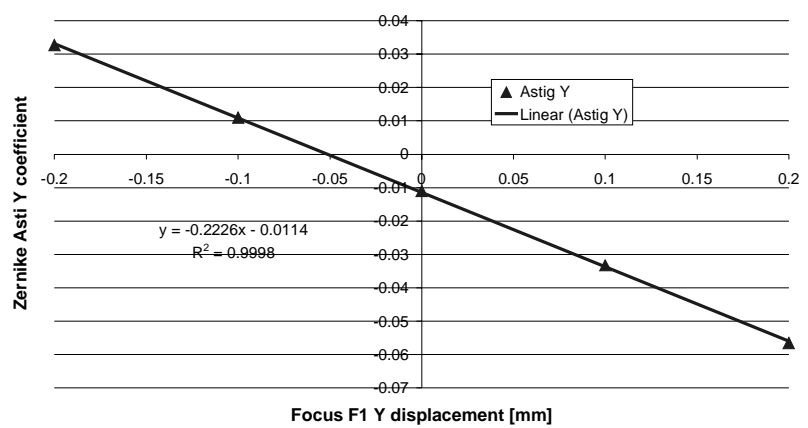

Figure 16: Evolution of the Zernike $Y$ astigmatism coefficient for different F2 Y positions. Same behaves for displacement of $F 2 \quad X$ for $X$ astigmatism Zernike coefficient

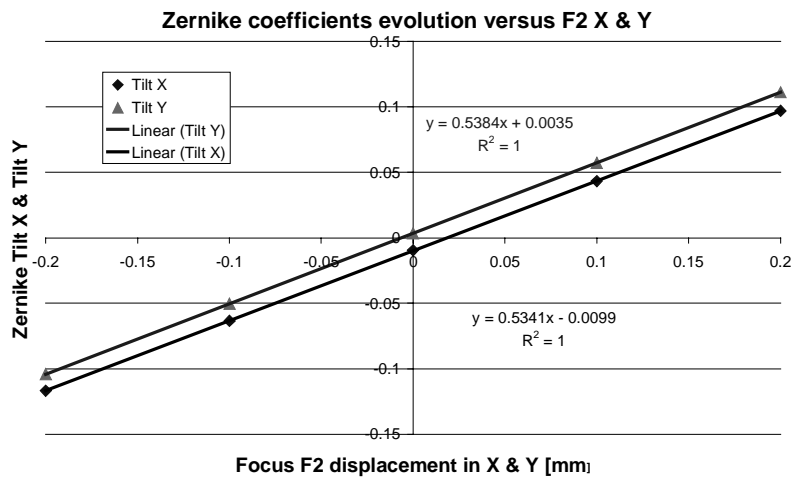

Figure 18: Evolution of the Zernike $X$ tilt and $X$ astigmatism coefficient for different $F 2 X$ positions. Same behaves for displacement of F2 $Y$ for $Y$ tilt and $Y$ astigmatism Zernike coefficient

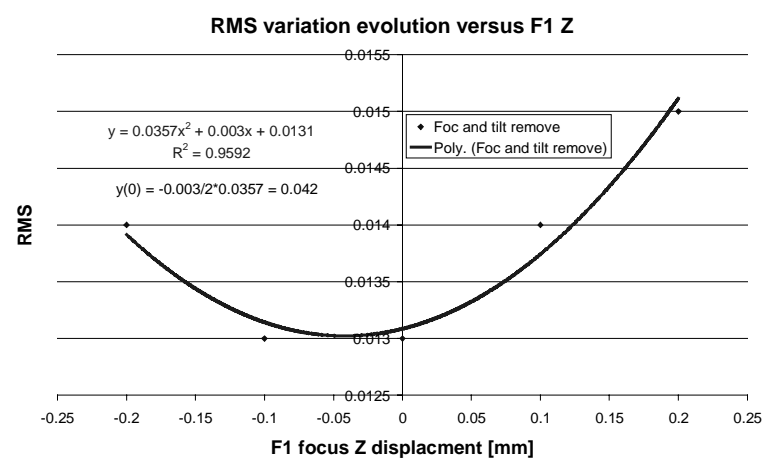

Figure 17: Evolution of the RMS coefficient for different Z F1positions

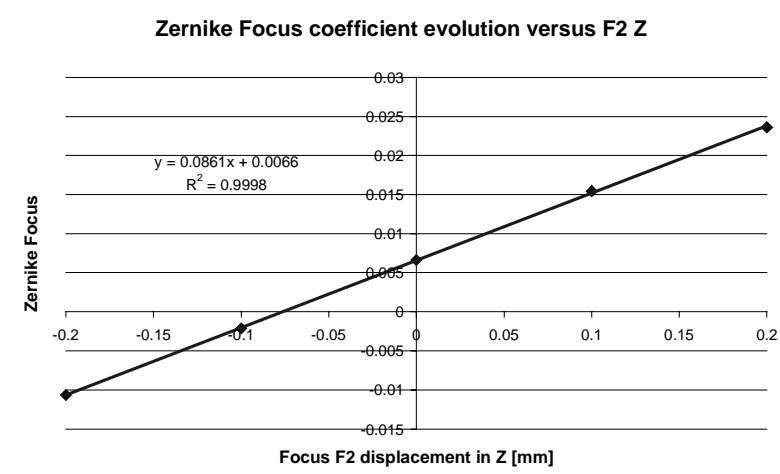

Figure 19: Evolution of the Focus Zernike coefficient for different $Z F 2$ positions.

\section{THE PROCEDURE APPLIED TO THE TEST SET-UP}

We applied the procedure of $\S 8$ to the real set-up. Once the antenna is integrated in the set-up (Figure 3), and that a preliminary alignment is achieved (fringes are observed on the interferometer Figure 20 and they can be unwrapped Figure 21 and OPD is computed Figure 22), the conditions are gathered to start the fine alignment procedure.

Typical results are given in the Figure 23 to Figure 26. It is nice to observe that the system behaves as simulated. The procedure as been tested by several operators and it appears to be robust. Each operator starts from different misaligned positions, but usually close (in a $500 \mu \mathrm{m}$ cube) to the best focus position. A repeatability (in term of the best focus positions) is achieved within $32 \mu \mathrm{m}$ RMS (60 $\mu \mathrm{m}$ PTV) in the worst case (F1Z), $17 \mu \mathrm{m}$ RMS (30 $\mu \mathrm{m}$ PTV) for F1Y, while all the other positions are determined within few $\mu \mathrm{m}$ RMS which is within the requirements The experimental data follows also well a linear behaviour. The computed correlation coefficients are always better than 0.9 and usually close to 0.99 . It appears also a good reproducibility of the linear slope between each alignment. 


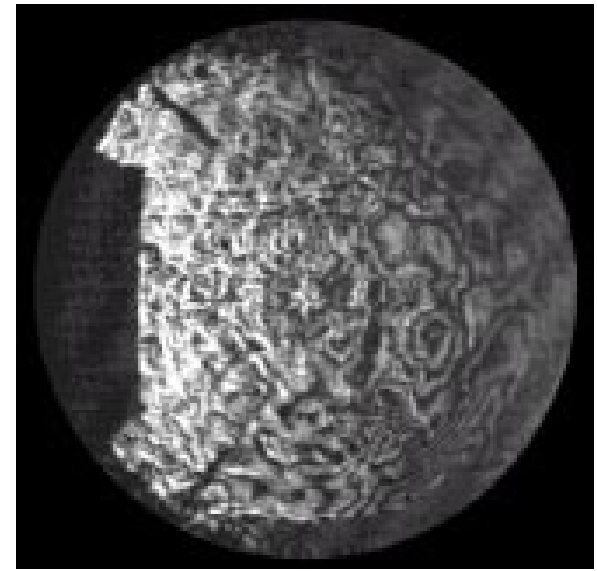

Figure 20 : Typical interferogram at $293 \mathrm{~K}$

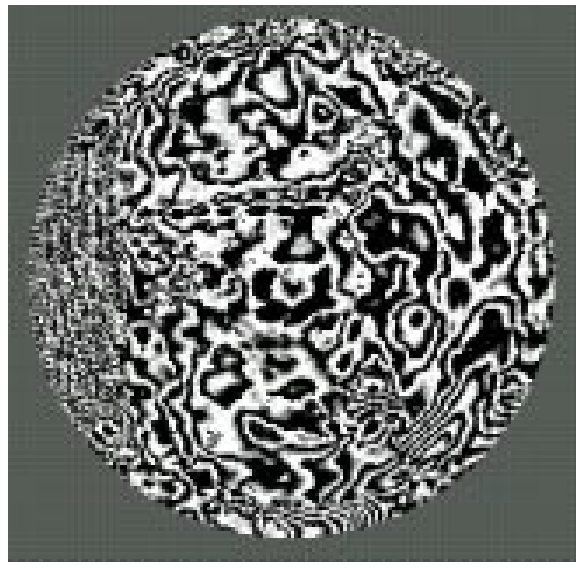

Figure 21: Wrapped phase computed from interferogram

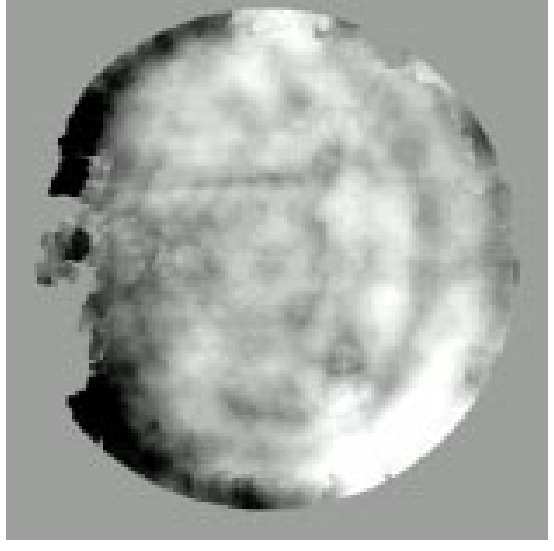
Figure 22: Computed OPD

$$
\text { . }
$$

Zernike Asti coefficients evolution versus F1 X \& Y

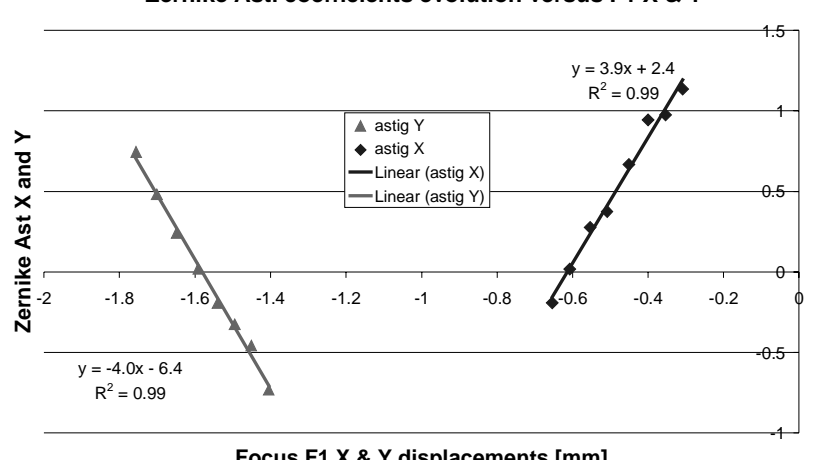

Focus F1 X \& Y displacements [mm]

Figure 23: Evolution of the Zernike $X$ and $Y$ astigmatism coefficient for different positions of $F 1 X$ and Y. Same slope is observed for both regression lines. The difference in sign comes from the experimental motor configuration.

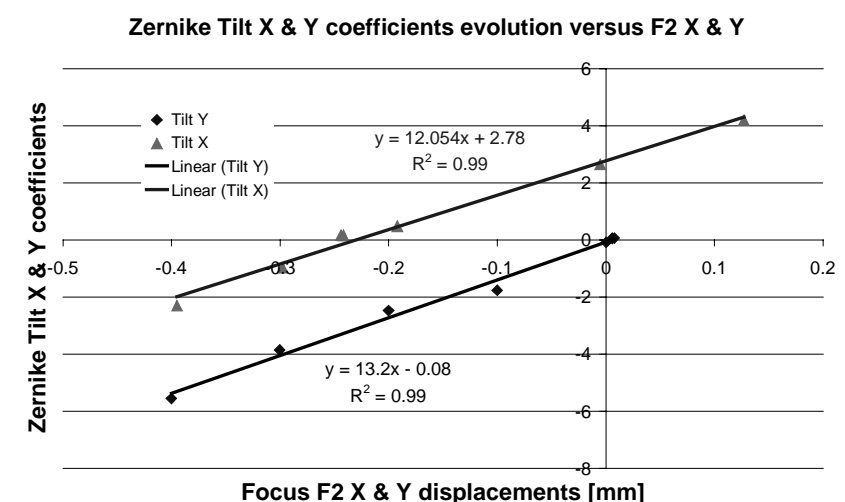

Figure 25: Evolution of the Zernike $X$ and $Y$ tilt coefficient for different positions of $F 2 X$ and $Y$. As it can be observed the slopes are close to each other.
RMS variation evolution versus $\mathrm{F} 1 \mathrm{Z}$

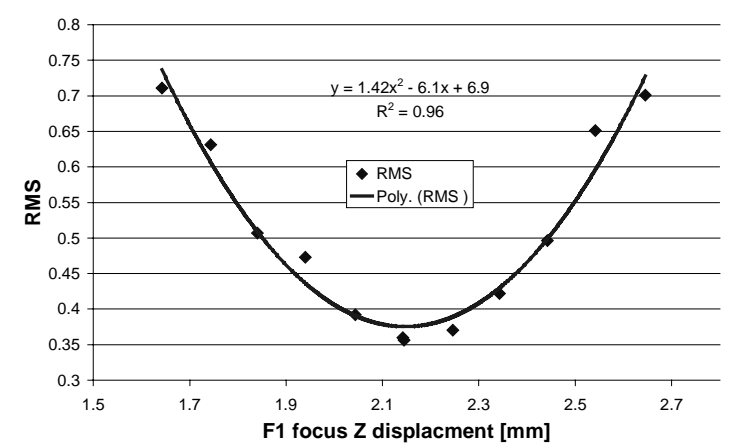

Figure 24: Evolution of the RMS coefficient for different Z F1positions.

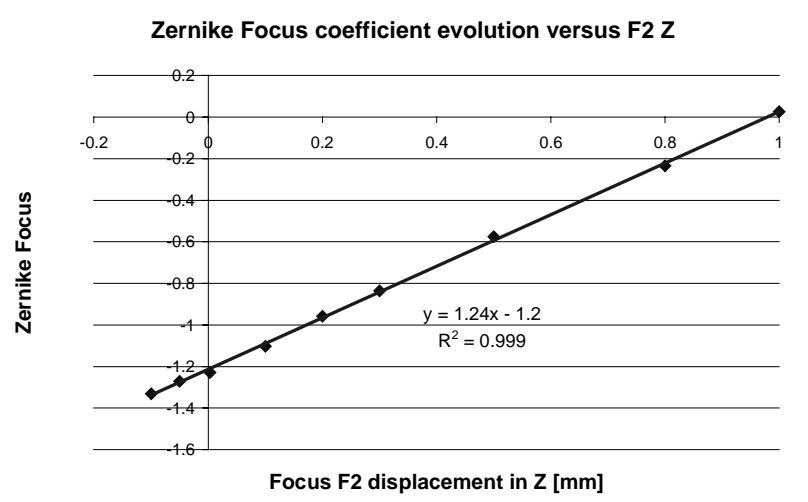

Figure 26: Evolution of the Focus Zernike coefficient for different Z F2 positions. 


\section{THE PROCEDURE APPLIED DURING THE CRYOGENIC TEST}

During the temperature transition, the temperature is stabilised to allow alignment at a fixed temperature.

The results of the presented procedure are shown in Figure 30 to Figure 33. It still looks to work correctly, however an accurate observation indicates that the linear correlation coefficients either they are still acceptable (close to 0.9 instead of 0.99) show that some problems are coming up. Additionally, there is a decrease in sensitivity mainly for the astigmatism terms (4 to 2.3). In fact, at cryogenic temperature, new surface figure error characteristics appear, which are inherent to the reflector technology [3]. Locally interferometric fringe density augments with a consequent loss in modulation and introduction of phase unwrapping errors. This indicates the limit of the method, but also of the PSI IR set-up.

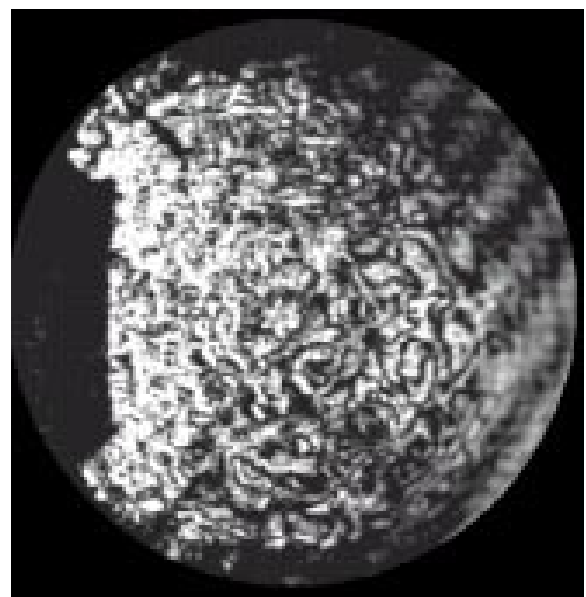

Figure 27 : Typical interferogram at $225 \mathrm{~K}$

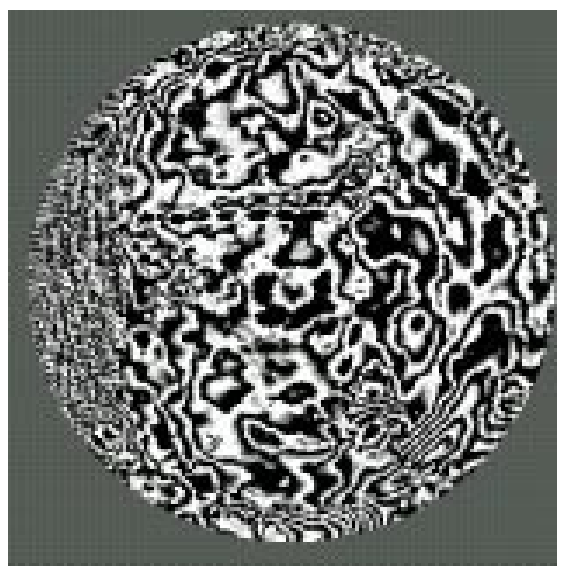

Figure 28: Computed interferogram from wrapped phase

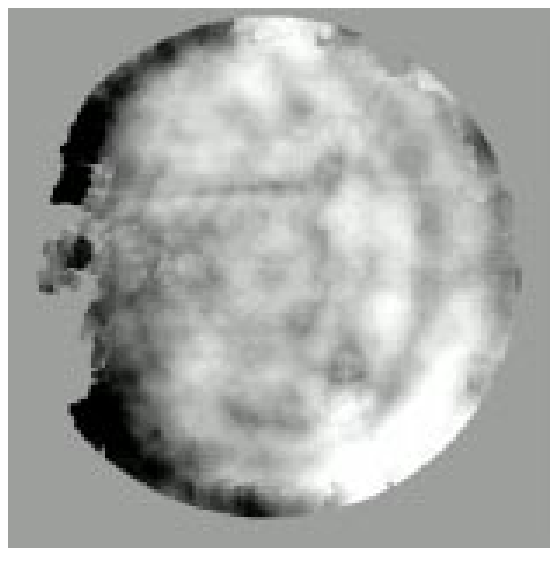

Figure 29: Computed OPD
Zernike Asti coefficients evolution versus F1 X \& Y

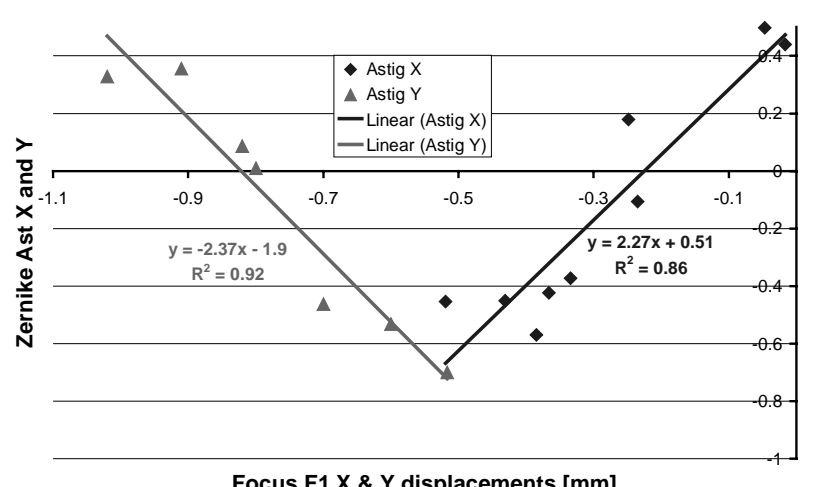

Figure 30: Evolution of the Zernike $X$ and $Y$ astigmatism coefficients for different FI $X$ and FI Y positions. Same slope is observed for both regression lines. The difference in sign comes from the experimental motor configuration.

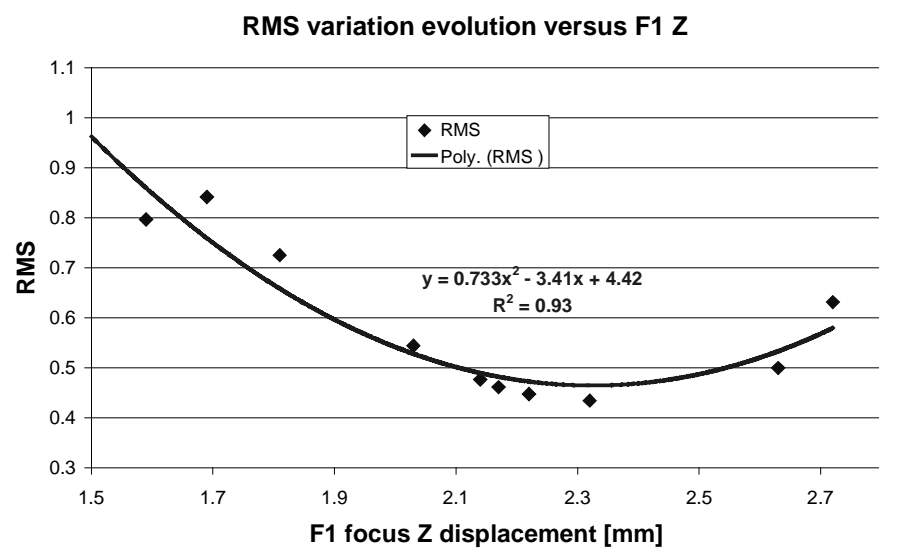

Figure 31: Evolution of the RMS coefficient for different Z F1 positions. 


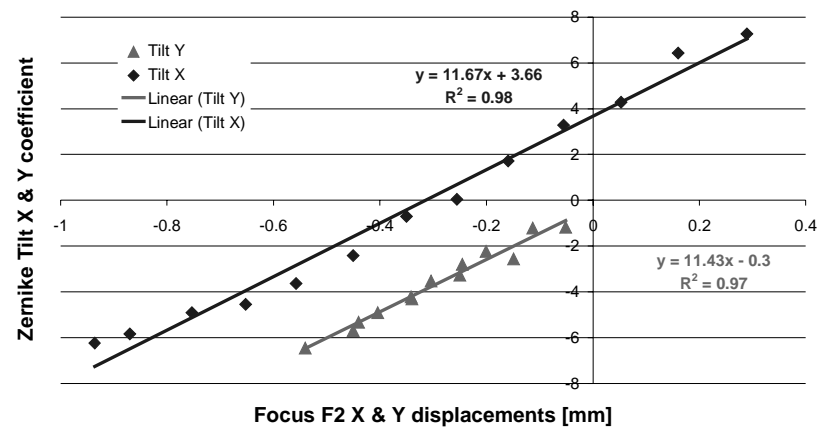

Figure 32: Evolution of the Zernike $X$ and $Y$ tilt coefficients for different $F 2 X$ and $F 2 Y$ positions.

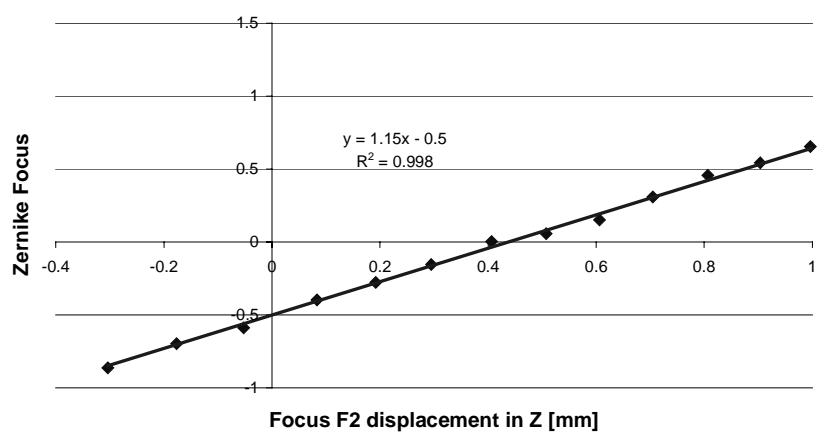

Figure 33: Evolution of the Focus Zernike coefficient for different $Z F 2$ positions.

\section{SUMMARY}

The different steps presented here above can be summarised. The Zernike polynomial sensitivity with respect to focus displacements is recapitulated in the table here after. In this table, the sensitivities are expressed in waves $(10.6 \mu \mathrm{m})$ per $\mathrm{mm}$. These information can help to speed up the alignment. Indeed, starting from an initial set of Zernike coefficient, it is possible to go straightforward (at least two steps) to a position close to the best focus and then start the described procedure. Another issue is to control the alignment and its reliability. When the sensitivities are changing with respect to the nominal values its indicates that something is going wrong. Several sources of troubles were faced. E.g. bad position of the mask which utilise not the correct part of the mirror, unwrapping errors, bad interferogram due to external mechanical perturbation. Thanks to these sensitivity slopes, any anomalies can be tackle very soon in the procedure. From the table here after it is easy to observed that the simulated world is more sensitive than the actual one. In the simulated case, the interferograms are correctly and entirely unwrapped. Actually the OPD is first computed and the interferogram is wrapped. In the practical case additional errors are coming in and the results are worst.

\begin{tabular}{|c|c|c|c|c|}
\hline Axes/Zernike & Ideal & Simulated & $300 \mathrm{~K}$ & $225 \mathrm{~K}$ \\
\hline Asti X & 5.5 & 5.7 & 3.9 & 2.27 \\
\hline Asti Y & 5.3 & 5.6 & -4 & 2.37 \\
\hline Tilt X & 13.7 & 13.3 & 12 & 11.5 \\
\hline Tilt Y & 13.7 & 13.5 & 13.2 & 11.8 \\
\hline Focus & 2.0 & 2.15 & 1.2 & 1.15 \\
\hline & & & & \\
\hline
\end{tabular}

Figure 34 : Table summarising the Zernike polynomial sensitivity with respect to foci displacements. The results are not the same as in the previous graphs, since the data are computed on different computers and with different settings. Additionally the units are not the same (mm, waves, motor steps, WFE, SFE...). This table reformulates it in the same units (Wave WFE /mm) for comparison purposes.

\section{CONCLUSION}

An unambiguous way to find the best focus position of an off axis ellipsoid is demonstrated. The proposed alignment is based on minimising the WFE RMS, and by sequential cancellation or at least minimising the dominant Zernike polynomial coefficients corresponding to tilt X, tilt Y, Astigmatism X, Astigmatism Y and Focus. The method has been demonstrated on an ASAP model for a perfect ellipsoid as well for a deformed one. The introduced deformations came from a $3 \mathrm{D}$ metrology measurements. This model takes into account all the lenses designed for the application as well as the corresponding displacements of the motors. In a second step, theory was applied to practice. In the actual world, the alignment is a long process, practically it takes about 4 hours to acquire, handle the data and put the lenses at the right 
places. Nevertheless, the process is quite reliable and repeatable within the requested specification (20 $\mu \mathrm{m}$ RMS) including the operator skills and harsh environmental conditions.

In our experiment, WFE measurements were made interferometrically, but nothing prevents to use other wavefront sensors. The procedure is device independent.

\section{Acknowledgements}

The authors are grateful to all the CSL operators for all the support they provided during this project.

This work was performed within the frame of ESA contract number 17913/03/NL/NB.

\section{References}

[1] Optical Shop Testing, D. Malacara, Wiley and Sons, January 1992.

[2] N. Ninane "On ground verification of high precision antenna structures", Report N RP-CSL-FIR-93006, 1993.

[3] Brian Catanzaro et al. "Optical Metrology for Testing an All-Composite 2-Meter Diameter Mirror" Proc. SPIE 4444, Aug 2001. 